\title{
Research on Evaluation System Construction and Empirical Analysis of Self-dependent Innovation Demonstration Areas Competitiveness: The Case of Three Demonstration Areas in Beijing, Shanghai, Wuhan
}

\author{
Na Zhang ${ }^{1,2}$, Ziwen Chen ${ }^{1,2}$ \\ ${ }^{1}$ School of Management, Wuhan University of Technology, Wuhan 430070, China \\ ${ }^{2}$ Center for Product Innovation Management of Hubei Province, Wuhan 430070, China
}

Keywords: Self-dependent Innovation Demonstration Area, Demonstration Area Comprehensive Competitiveness,"The four-dimensional CIAE" Evaluation System, Composite Relation Entropy Matter-element Model.

\begin{abstract}
Self-dependent innovation demonstration area is the demonstrating and leading zone in the development of the self-dependent innovation and high-tech industry promotion in China, whose competitiveness performance will determine the developing direction of our country's innovation level. This paper constructs a self-dependent innovation demonstration area competitiveness evaluation model with the evaluation index system called "four-dimensional CIAE" reflecting the core functions of self-dependent innovation demonstration including carrier support, Independent innovation, agglomeration-radiation and external expansion, utilizes the composite relation entropy matter-element model and conducts a comparative analysis of the performances and the characteristics of three large demonstration areas' comprehensive competitiveness, which provides a scientific and effective evaluation tool for the national self-dependent innovation demonstration area construction.
\end{abstract}

\section{Introduction}

The self-dependent innovation demonstration area, approved by the State Council in China acting as a role of exploring experience, making demonstration in promoting self-dependent innovation and high technology industry development pilot area, imposes significant effect on driving the innovation engine development and speeding up the shift of the mode of our country's economic development.

Foreign theoretical and industrial circles design the high-tech zone competitiveness evaluation system from different angles. On behalf of the main achievement are as follows: Bruno and Tyebjee designed the corresponding evaluation index system according to venture capital, policy tilt, entrepreneur ability, convenient traffic and other 12 factors ${ }^{[1]}$. Makecki evaluated the circumstance of high-tech zone according to the fluidity of research personnel, the fluidity of venture capital, government support and the like 8 factors. Silicon Valley Network published annually index since 1995 to the present, which was according to 5 aspects of human resources, innovation economy, the living place and regional management with a total of 82 measures $^{[2-3]}$. The domestic scholar Shouqing $\mathrm{Fu}$ elaborated 5 stages and corresponding features of the demonstration area with the example of Zhongguancun demonstration area, and summarized regional innovation networks and industrial ecological environment were two key factors ${ }^{[4]}$.Shuhua $\mathrm{Hu}$ analyzed the internal relationships and operational mechanism of 12 innovative elements of demonstration area in system dynamics ${ }^{[5]}$.Jialong Xie discussed unbalance degree and change trend of high-tech zone in our country on the basis of international foreign exchange earnings, innovation, social contribution, support with a total of 21 indicators evaluation system ${ }^{[6]}$. 


\section{Comprehensive Competitiveness Evaluation System Building of Three Demonstration Areas}

\subsection{Construction of “The Four-three Structure” Mode and Index Selection}

This paper takes the theoretical achievements of Jialong Xie for reference. The index system framework is designed from four dimensions (hereinafter referred to as the "The Four-dimensions CIAE”), such as Carrier Support, Independent Innovation, Accumulation-Radiation and External Expansion $^{[6]}$.

Table 1 “The Four-dimensional CIAE” Competitiveness Evaluation Index

\begin{tabular}{|c|c|c|}
\hline first level index & Second level index & third level index \\
\hline \multirow{14}{*}{$\begin{array}{l}\text { Carrier Support } \\
\text { B1 }\end{array}$} & \multirow{8}{*}{ Hard carrier C1 } & D1:enterprise average \\
\hline & & D2:employee scale \\
\hline & & D3:number of universities and research institutions \\
\hline & & D4:asset-liability ratio \\
\hline & & D5:geographical position \\
\hline & & D6 :number of Productivity promotion center \\
\hline & & D7:the total of Science and technology incubator \\
\hline & & D8 :Science and technology incubation space \\
\hline & \multirow{6}{*}{ Soft carrier C2 } & D9 policy support dynamics \\
\hline & & D10:campus culture of innovation \\
\hline & & D11: local laws completeness \\
\hline & & D12 :professional quality of service \\
\hline & & D13 :venture capital support \\
\hline & & D14: basis supporting environment \\
\hline \multirow{14}{*}{$\begin{array}{l}\text { independent } \\
\text { innovation B2 }\end{array}$} & \multirow{6}{*}{ Innovation input C3 } & D15:average stock of R\&D funds \\
\hline & & D16 :the intensity of R\&D spending \\
\hline & & D17 :activities of science and technology personnel \\
\hline & & D18 :talent input structure \\
\hline & & D19:employees' level of education \\
\hline & & $\begin{array}{l}\text { D20 :the activities of science and technology personnel per capita } \\
\text { R\&D funds }\end{array}$ \\
\hline & \multirow{8}{*}{ Innovation output C4 } & D21 :technology income scale \\
\hline & & D22 :income outcome structure \\
\hline & & D23 :income R\&D funding to create technology \\
\hline & & D24 :new product sales ratio \\
\hline & & D25 :patent weighted quantity \\
\hline & & D26 :the relative amount of enterprise incubator graduation \\
\hline & & D27 :profitability \\
\hline & & D28 :the number of independent well-known brands \\
\hline \multirow{9}{*}{$\begin{array}{l}\text { Agglomeration \& } \\
\text { Radiation B3 }\end{array}$} & \multirow{4}{*}{$\begin{array}{l}\text { Accumulate strength } \\
\quad \text { C5 }\end{array}$} & D29 :human concentration of high-technology \\
\hline & & D30 :capital element concentration \\
\hline & & D31 :space gathered density \\
\hline & & D32 :science and technology incubation density \\
\hline & \multirow{5}{*}{$\begin{array}{l}\text { Radiation driven } \\
\quad \text { C6 }\end{array}$} & D33 :economic driving degree \\
\hline & & D34 :export pulling force \\
\hline & & D35 :employment absorption force \\
\hline & & D36 :tax contribution \\
\hline & & D37 :environment improving degree \\
\hline \multirow{8}{*}{$\begin{array}{c}\text { External } \\
\text { Expansion B4 }\end{array}$} & \multirow{4}{*}{$\begin{array}{l}\text { Foreign exchange } \\
\text { earning level } \\
\text { C7 }\end{array}$} & $\begin{array}{c}\text { D38 export scale } \\
\end{array}$ \\
\hline & & D39 per capita export \\
\hline & & D40 export growth \\
\hline & & D41 degree of dependence on export \\
\hline & \multirow{4}{*}{$\begin{array}{c}\text { Strength of investment } \\
\text { and capital attracting } \\
\text { C8 }\end{array}$} & D42:the degree of park openness \\
\hline & & D43 :the actual use of foreign capital scale \\
\hline & & D44:per capita use of pinch \\
\hline & & D45 degree of utilization of foreign capital \\
\hline
\end{tabular}


Through theory selection and empirical selection, evaluation criterions of the competitiveness form index system including four dimensions and three layers. This index system covers 4 first level indexes, 8 second level indexes and 45 third level indexes, and combines quantitative indexes and qualitative indexes, absolute indexes and relative indexes. The design is an active extension and bold attempt based on the existing research results, and that also ensures the objectivity and feasibility of the index system.

\subsection{Evaluation Model Building of the Competitive Comprehensive}

Due to the statistical caliber normalization of demonstration areas is relatively poor, and evaluation indexes contain a lot of qualitative indexes, leading to not explicitly give weight information of evaluation indexes. In order to improve the objectivity of evaluation process, the paper adopts relative entropy to determine the weight coefficient of each index.

First, calculate the grey correlation coefficient of $\underset{\sim m n}{R}$. The reference sequence $Y_{0}=\left\{y_{1}, y_{2}, \cdots, y_{n}\right\}^{T}\left(y_{j}=\max _{1 \leq i \leq m} \mu_{i j}, j=1,2, \cdots, n\right)$ is established. Compare sequences and reference sequences together make up the grey correlation @ $G R F=\left[Y_{0} \underset{\sim m n}{R}\right]$, thus to get diversity sequence $\Delta_{i}=\left|Y_{0}-Y_{i}\right|$. Then grey relational coefficient of $C_{j}$ is expressed as

$$
\zeta_{i j}=\frac{\min _{i} \min _{j} \Delta_{i}+\rho \max _{i} \max _{j} \Delta_{i}}{\left|\mu_{i j}-y_{j}\right|+\rho \max _{i} \max _{j} \Delta_{i}}(1)
$$

The $\rho$ in the formula is distinguish coefficient, normally $\rho=0.5$, in order to weaken distortion of correlation coefficient of evaluation indexes, because the biggest absolute difference is too large.

Then, calculate the entropy of evaluation index. Because entropy is a function of measurement uncertainty in information theory, entropy value is bigger, the greater the corresponding information. So the entropy of $C_{j}$ is

$$
F_{j}=-(\ln m)^{-1} \sum_{i=1}^{m} \frac{\zeta_{i j}}{\sum_{i=1}^{m} \zeta_{i j}} \ln \frac{\zeta_{i j}}{\sum_{i=1}^{m} \zeta_{i j}},(i=1,2, \cdots, m ; j=1,2, \cdots, n)
$$

Finally, determine the weight coefficient of evaluation index. According to the deviation degree $k_{j}=1-F_{j}$, get the weight coefficient of $C_{j}$ is $\omega_{j}=k_{j} / \sum_{j=1}^{n} k_{j}$. And build a composite matter-element of indexes' weight of the competitiveness evaluation of demonstration zones:

$$
R_{\omega j}=\left[\begin{array}{ccccc} 
& C_{1} & C_{2} & \cdots & C_{n} \\
\omega_{j} & \omega_{1} & \omega_{2} & \cdots & \omega_{n}
\end{array}\right] \text { (3) }
$$

(4) Calculate the evaluation results of comprehensive competitiveness

Composite relation entropy matter-element $\underset{\sim m H}{R}$ of the competitiveness evaluation is made by $\underset{\sim m n}{R}$ and $R_{\omega j}$.

$$
\begin{aligned}
& \underset{\sim m H}{R}=\left[\begin{array}{ccccc} 
& M_{1} & M_{2} & \cdots & M_{m} \\
H_{i} & H_{1} & H_{2} & \cdots & H_{m}
\end{array}\right]
\end{aligned}
$$

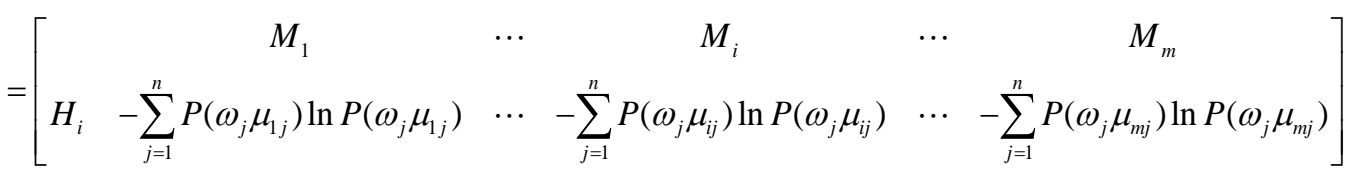

In this formula, $P\left(\omega_{j} \mu_{i j}\right)=\omega_{j} \mu_{i j} / \sum_{j=1}^{n} \omega_{j} \mu_{i j}, i=1,2, \cdots, m ; j=1,2, \cdots, n$, measure value of the competitiveness evaluation of $i$ is $H_{i}$. And more value of the $H_{i}$, comprehensive competitiveness the better. Thereby comprehensive competitiveness of three demonstration zones is 
ranked by the value of $H_{i}$.

\section{Comparative analysis of comprehensive competitiveness of Demonstration Area}

Combining with demonstration area's competitiveness evaluation structure of "four dimensional CIAE", comparative analysis of performance and characteristics of three demonstration areas' competitiveness is conducted from the " $4+1$ " dimensions of carrier support, self-dependent innovation, the development of accumulated-radiation, external expansion and comprehensive competitiveness.

Table2 comprehensive competitiveness evaluation measure value about "The Four-dimensional CIAE” of Three Demonstration Areas

\begin{tabular}{ccccccc}
\hline Demonstration Area & Zhongguancun & sort & Zhangjiang & sort & $\begin{array}{c}\text { East } \\
\text { Lake }\end{array}$ & Sort \\
\hline Carrier Support B1 & 0.7390 & 1 & 0.6207 & 2 & 0.4127 & 3 \\
\hline Hard carrierC1 & 0.8275 & 1 & 0.5134 & 2 & 0.4672 & 3 \\
\hline Soft carrierC2 & 0.6485 & 2 & 0.7304 & 1 & 0.3570 & 3 \\
\hline \hline Independent Innovation B2 & 0.8375 & 1 & 0.4460 & 3 & 0.4820 & 2 \\
\hline Innovation input C3 & 0.7200 & 1 & 0.4888 & 3 & 0.5938 & 2 \\
\hline Innovation output C4 & 0.9157 & 1 & 0.4176 & 2 & 0.4076 & 3 \\
\hline Agglomeration Radiation B3 & 0.9463 & 1 & 0.3854 & 3 & 0.4508 & 2 \\
\hline Accumulate strength C5 & 0.9030 & 1 & 0.3547 & 3 & 0.4650 & 2 \\
\hline Radiation driven C6 & 0.9857 & 1 & 0.4133 & 3 & 0.4379 & 2 \\
\hline External Expansion B4 & 0.4383 & 3 & 0.9182 & 1 & 0.4670 & 2 \\
\hline Foreign exchange earning level C7 & 0.4392 & 3 & 0.8412 & 1 & 0.5230 & 2 \\
\hline Strength of investment attractinC8 & 0.4373 & 2 & 1.0000 & 1 & 0.4076 & 3 \\
\hline \hline Comprehensive Competitiveness & 0.7643 & 1 & 0.5637 & 2 & 0.4528 & 3 \\
\hline 3.1 Comparative analysis of Carrencen
\end{tabular}

\subsection{Comparative analysis of Carrier Support}

According to the calculated result which can be found in table 2, the carrier support of Beijing Zhongguancun (0.7390) and Shanghai Zhangjiang (0.6270) have similar performance, more outstanding than the others. The hard carrier of Zhongguancun (0.8275) and the soft carrier of Zhangjiang (0.7304) are in the first place of the three demonstration areas. By comparison, the construction level of hard carrier and soft carrier of Wuhan East Lake are the lowest. In the field of hard carrier, Wuhan East Lake not only has significant congenital disadvantage in the location (central inland), but also shows weakness in many indexes (enterprise average $\mathrm{D}_{1}$ and employee scale $\mathrm{D}_{2}$ ). Similarly, it is also weak in the area of soft carrier support formed by government departments, agencies, and venture capital firms.

\subsection{Comparative analysis of Self-dependent Innovation Ability}

According to the calculated result which can be found in table 2, the innovation input $(0.7200)$ and the output (0.9157) of Beijing Zhongguancun have obvious leading advantage, much higher than the other two demonstration areas and the score of the whole competition condition of the self-dependent innovation B2 is almost twice as that of Wuhan East Lake. The calculated structure of Wuhan East Lake is close to Shanghai Zhangjiang, which is 0.4460 and 0.4820 respectively. In innovation output C4 area and the scores of most indexes of Wuhan East Lake are slightly higher than the Shanghai Zhangjiang, showing that Wuhan East Lake obtains higher innovation output in the condition of lower scale of innovation investment and has stronger transfermation ability of science and technology.

\subsection{Comparative analysis of Accumulating-radiation Force}

According to the calculated result which can be found in table 2, Beijing Zhongguancun area has the best accumulating-radiation effect $(B 3=0.9463)$, even higher than the total score of Shanghai Zhangjiang and Wuhan East Lake. As a central leader in science and technology innovation and industry upgrading, Wuhan shows better radiation intensity $(C 5=0.4650)$ and the radiation drive (C6 $=$ 0.4379). In addition, its score of accumulation of radiation B3 is slightly higher than the 
Shanghai Zhangjiang, ranking the second. At the same time, it contributes $45.43 \%$ for export-led region relying on advantages of technology and products in the field of optoelectronic information industry.

\subsection{Comparative analysis of of External Expansion Force}

According to the calculated result which can be found in table 2, the various indexes of the external expansion force of Shanghai Zhangjiang $B_{4}$ ranks the first, much higher than the others. By contrast, the external expansion trends of Beijing Zhongguancun and Wuhan East Lake are weak, but Wuhan East Lake stands firm in the international market by virtue of optoelectronic information technology (Changfei, Fenghuo, etc.), and has higher foreign exchange earning level than that of Beijing Zhongguancun. Through strengthening export orientation of science and technology products and supporting the area in aspects such as taxation, financing and technology, many indexes (export earnings growth D40, average use of resource D44, and degree of utilization of foreign capital D45) of Wuhan East Lake shows a rising trend. Although there is still a gap compared with mature coastal high-tech zone at present, the external expansion of East Lake will be expanded with the rise of the Yangtze River economic belt.

\section{Conclusion}

Results show that the Beijing Zhongguancun demonstration zone has stronger competitive advantages than Zhangjiangin in all aspects except the external expansion, especially self-dependent innovation B2 and accumulation-radiation B3, which has the strongest comprehensive competitiveness. The comprehensive competitiveness of Shanghai Zhangjiang demonstration zone ranks the second, but still much weaker than the Zhongguancun demonstration zone. Having the unique geographical advantage, Zhangjiang demonstration area's external expansion B4 is outstanding, but self-dependent innovation B2 and accumulation-radiation B3 are poor. Comprehensive competitiveness of Wuhan East Lake demonstration area ranks the last and the carrier support B1 level is low, especially soft carrier support. However, in recent years, its input intensity of various innovative elements has increased, external expansion has been increasing apparently, and export earning has been growing by years. Additionally, Wuhan East Lake is advantageous in areas of the self-dependent innovation B2 and accumulation-radiation B3, compared with Zhangjiang demonstration area.

\section{Acknowledgment}

This paper is supported by National Science Foundation of China (71373199) and the Fundamental Research Funds for the Central Universities (WHUT 2015-zy-039, 2014-zy-054, 2014-zy-050, 2014-Ib-003)

\section{Reference}

[1]Bruno A.V, Tyebjee T. T. The Environment for Entrepreneurship. Englewood Cliffs Prentice-Hall, NJ.1982.

[2]Malecki E.J. The R\&D location decision of the firm and creative regions-A survey. Technovation,1987, p. 205-222.

[3]Malecki E.J, Nijkamp P. Technology and regional development: some thoughts on policy. Environment and Planning C: Government and Policy, 1983,p. 383-399.

[4]Shouqing $\mathrm{Fu}$, et al. Study on regional innovation network and the technology industry ecological environment interactive mechanism: the case of Haidian science park of Zhongguancun Hi-tech zone. Management world, 2010,p. 8-13,27.

[5]Shuhua Hu, Song Wang, Henjin Deng.Study on the development of national independent innovation demonstration park based on four-three structure model. Science \& Technology Progress and Policy, 2011,p. 29-32.

[6]Jialong Xie, Shuhua Hu. Design and application of the selection system for national indigenous 
innovation demonstration zone. China Soft Science, 2013,p. 67-79. 